\title{
Estado actual del diagnóstico y manejo de la pancreatitis crónica
}

\section{The current state of diagnosis and management of chronic pancreatitis}

\author{
Lázaro Antonio Arango M., MD; Claudia Patricia Díaz T., MD²; Carlos Andrés Caicedo Q., MD³; Camilo Ángel Rodríguez, MD
}

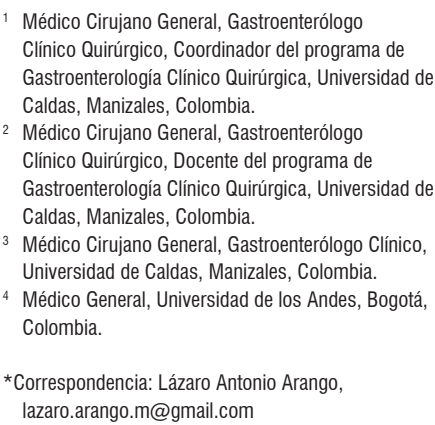

3 Médico Cirujano General, Gastroenterólogo Clínico, Universidad de Caldas, Manizales, Colombia.

${ }^{4}$ Médico General, Universidad de los Andes, Bogotá, Colombia.

*Correspondencia: Lázaro Antonio Arango, lazaro.arango.m@gmail.com

Fecha recibido: $\quad$ 14/10/18 Fecha aceptado: $04 / 09 / 19$

\begin{abstract}
Resumen
La pancreatitis crónica (PC) es una condición inflamatoria que lleva a fibrosis, con destrucción y compromiso del parénquima pancreático y de los ductos. Estos cambios permanentes pueden llevar a alteración de la función pancreática tanto exocrina como endocrina, estenosis biliares y pancreáticas, que llevan a su vez a otras consecuencias, como la formación de pseudoquistes, incluso incrementa la posibilidad de desarrollar cáncer de páncreas. La principal característica clínica de estos pacientes es el dolor, el cual altera de forma importante su calidad de vida. Para diagnosticar la PC contamos con pruebas funcionales, las cuales se pueden dividir en directas o indirectas, y pruebas de estructura pancreática.

El gran reto de estos métodos consiste en diagnosticar la enfermedad en estadios iniciales, donde la aproximación diagnóstica es más difícil por la sutileza de los cambios. Una vez diagnosticada la PC, el manejo también debe ser escalonado; este se puede dividir a su vez en manejo médico, como paso inicial, manejo endoscópico y manejo quirúrgico o, en los casos más difíciles, la combinación de estos. El objetivo es manejar y entender al paciente y su enfermedad como un todo, con el fin de proporcionarle la mejor calidad de vida posible al paciente. A continuación, se presenta una revisión enfocada en el diagnóstico y manejo de esta entidad a la luz de la evidencia actual disponible.
\end{abstract}

\section{Palabras clave}

Pancreatitis crónica, diagnóstico, tratamiento.

\section{Abstract}

Chronic pancreatitis (CP) is an inflammatory condition that leads to fibrosis, damage, and even destruction of the pancreatic parenchyma and ducts. These permanent changes can alter pancreatic exocrine and endocrine functioning, cause biliary and pancreatic stenosis, lead to formation of pseudocysts and even increase the possibility of developing pancreatic cancer. The main clinical characteristic is pain which significantly alters quality of life. To diagnose the $\mathrm{CP}$, we have direct and indirect functional tests and the pancreatic structure test.

The great challenge of these methods is early diagnosis, but this is difficult due to the subtlety of changes. Once CP is diagnosed, management must be staggered. Medical management is the initial step which can be followed by endoscopic management, surgical management, and for the most difficult cases a combination of these. The goal is to manage and understand the whole patient and illness to provide the best possible quality of life. This review article focuses on CP diagnosis and management in light of the currently available evidence.

\section{Keywords}

Chronic pancreatitis, diagnosis, treatment. 


\section{INTRODUCCIÓN}

La pancreatitis crónica (PC) es un proceso inflamatorio del páncreas, en el cual, el parénquima de la glándula es alterado y reemplazado por tejido fibroso, lo que resulta en cambios permanentes a nivel del ducto y del parénquima pancreático y lleva a disfunción endocrina y exocrina ( 1 , 2). La principal característica clínica es el dolor, el cual está presente en la mayoría de los pacientes, junto a otras características clínicas, las cuales dependen del grado de disfunción endocrina y exocrina pancreática (3).

En esta revisión nos centraremos en los métodos diagnósticos y en las diferentes modalidades terapéuticas actuales para esta entidad. Es importante entender a la enfermedad como un todo y tratar de manejar cada caso de una forma integral, de esto dependerán los resultados de cada paciente $y$, por consiguiente, de mejorar su calidad de vida.

\section{DIAGNÓSTICO}

La PC avanzada se diagnostica más fácilmente, comparada con la enfermedad en su estadio leve o moderado, en donde dicho diagnóstico se convierte en un desafío. Para el diagnóstico de esta entidad hay que tener en cuenta los síntomas clínicos del paciente, las pruebas de función pancreática y la interpretación de las imágenes, como la resonancia magnética (RM) y la ultrasonografía endoscópica (USE). Las pruebas para el diagnóstico se pueden clasificar en funcionales y estructurales (evalúan el parénquima, la anatomía del ducto o ambos). A su vez, las pruebas funcionales las podemos clasificar en directas e indirectas, teniendo estas últimas más capacidad diagnóstica cuando la enfermedad se encuentra en estadios más avanzados, pero con menor sensibilidad de detección para la enfermedad temprana o con compromiso moderado $(2,4)$.

\section{PRUEBA DE FUNCIÓN PANCREÁTICA}

\section{Prueba directa o invasiva}

Generalmente es poco usada, casi exclusiva de centros de investigación debido a lo dispendioso de su realización. Esta prueba consiste en la medición de bicarbonato del aspirado duodenal después de la estimulación con secretina o colecistocinina (CCK). Se considera compatible con disfunción pancreática exocrina cuando los niveles de bicarbonato medido son inferiores a $75 \mathrm{mEq} / \mathrm{L}$ (5). Esta prueba tiene una alta sensibilidad y especificidad, pero por ser invasiva y de alto costo, su uso es limitado en la práctica clínica (6).

\section{Prueba indirecta o no invasiva}

Estas pruebas no requieren una estimulación hormonal de la secreción pancreática directa. Su valor diagnóstico es limitado por la baja sensibilidad y especificidad que tienen para detectar alteraciones, sobre todo al inicio de la enfermedad (3). Entre estas pruebas contamos con tripsinógeno sérico, medición de grasa fecal, elastasa fecal y prueba respiratoria. La medición de la función endocrina usando métodos tradicionales, como la hemoglobina glucosilada, glucemia en ayunas o prueba de tolerancia oral a la glucosa, no ha sido bien caracterizada para el diagnóstico de la pancreatitis crónica, por lo que se requieren más estudios en este aspecto (7).

La medición sérica de tripsinógeno, con niveles inferiores a $20 \mathrm{ng} / \mathrm{mL}$, es específica para PC avanzada. La medición de grasa fecal es otra prueba indirecta, que consiste en cuantificar la grasa excretada durante 72 horas en un paciente que tuvo una ingesta limitada de $100 \mathrm{~g} /$ día por al menos 3 días antes de la prueba. El hallazgo de más de $7 \mathrm{~g}$ de grasa/día es diagnóstico de malabsorción. Tiene sensibilidad limitada, al igual que el tripsinógeno sérico, ya que en los pacientes con enfermedad leve o moderada no se encuentra alterada. La elastasa fecal con niveles superiores a $200 \mu \mathrm{g} / \mathrm{g}$ de materia fecal representa una baja posibilidad de insuficiencia pancreática exocrina (8).

La prueba respiratoria con triglicéridos (TGC) marcados con carbono 13, usado para el diagnóstico de la insuficiencia pancreática exocrina en pacientes con $\mathrm{PC}$, tiene una sensibilidad y especificidad del $92 \%$ y $91 \%$, respectivamente. Esta posee una tasa acumulativa de recuperación menor del $29 \%$, tomada a las 6 horas luego de la administración oral de $250 \mathrm{mg}$ de TGC, marcada junto a una comida con $16 \mathrm{~g}$ de grasa, esta indica insuficiencia pancreática exocrina (9).

\section{Prueba de estructura pancreática}

Dentro de los métodos diagnósticos por imágenes para el estudio estructural del páncreas en la PC encontramos el uso de la radiografía simple de abdomen. Este método tiene una muy baja sensibilidad, dado que cuando se observan calcificaciones pancreáticas (características de la PC) la enfermedad ya está muy avanzada. En el pasado se consideraba la colangiopancreatografía retrógrada endoscópica (CPRE) como el método definitivo para valorar los cambios del conducto pancreático con el uso de los criterios de Cambridge para el diagnóstico de la PC. Actualmente, este método fue reemplazado por otros, dado su naturaleza invasiva y las complicaciones asociadas con la CPRE (4).

La tomografía computada (TC) de abdomen está indicada en primera línea para el algoritmo diagnóstico en 
pacientes con sospecha de $\mathrm{PC}$, por su fácil accesibilidad y naturaleza no invasiva, teniendo un bajo rendimiento diagnóstico en PC leve a moderada; por esto, ante hallazgos no concluyentes en la TC se indicaría la realización de RM y de colangiorresonancia magnética (CPRM), ya que esta es superior a la TC para detectar cambios incipientes, tanto parenquimatosos como ductales, lo que mejora la sensibilidad en el diagnóstico de la PC temprana (10).

El uso de la secretina aumenta el potencial diagnóstico de la CPRM, ya que permite mejorar la valoración ductal y también la secreción pancreática hacia el duodeno. Todas estas imágenes, junto con la ecografía abdominal, nos permiten evaluar la presencia de cambios ductales, agrandamiento pancreático, calcificaciones y colecciones peripancreáticas (11).

Hoy por hoy, la USE es el método diagnóstico con mayor sensibilidad, la cual nos permite evaluar tanto el parénquima pancreático como el sistema ductal y tiene la capacidad de detectar cambios tempranos y tardíos en la PC (12-15). La finalidad de la USE consiste en evaluar alteraciones del parénquima y del ducto pancreático; además de intentar, por medio de criterios encontrados, hacer el diagnóstico en períodos tempranos.

Si se hace una revisión de la literatura faltan datos que soporten un método de clasificación y diagnóstico de la pancreatitis crónica por medio de criterios. Existen muchas clasificaciones y criterios, como los de Lees-Wiersema, Milwaukee, japonés y Rosemont, que evalúan en el ducto la presencia de dilatación, irregularidad, realce del mismo, visualización de conductos secundarios y cálculos en su interior; y en el parénquima focos hiperecoicos, bandas, lobularidad y quistes, que permiten clasificar estos hallazgos en definitivos o consistentes, sugestivos, indeterminados o no concluyentes (normales) de PC (13-16).

Recientemente apareció el importante concepto de los cambios mínimos en la endosonografía, que no llenan criterios para diagnosticar pancreatitis y su importancia. Esto aparece muy bien expuesto en el artículo de Sheel, en el que se describe el valor diagnóstico de los criterios japoneses y de Rosemont en pacientes con pancreatitis crónica indeterminada, sugestiva, posible o temprana (16). De lo dicho, es importante que no siempre se encuentran criterios que expliquen lo síntomas del paciente, que una gran mayoría de enfermos tienen dolor y signos típicos, con imágenes normales, en los que se puede ver en la USE algunos cambios discretos. En estos pacientes es importante valorar la historia, la parte social con la ingesta de alcohol y el consumo de cigarrillo, herencia familiar $y$, si se puede, estudios genéticos y hacer seguimiento endosonográfico por lo menos por 30 meses, observando quienes viran a una verdadera pancreatitis crónica o quienes sanan, así como la mejoría en los cambios endosonográficos. Asimismo, luego de la pancreatitis aguda se debe hacer seguimiento de estos pacientes en el mismo tiempo para determinar quiénes harán la enfermedad (16).

En la endosonografía es importante determinar cuáles son los criterios que más nos pueden ayudar para el diagnóstico. En el año 1993, Wiersema y colaboradores describieron una escala de 9 criterios diagnósticos. Estos son (17):

- Focos hiperecoicos

- Tractos fibrosos

- Lobularidad

- Quistes

- Calcificaciones

- Dilatación de ramas secundarias

- Irregularidad del conducto pancreático

- Paredes hiperecogénicas del Wirsung.

A todos estos criterios le dieron el mismo valor diagnóstico, pero no se ha encontrado un punto de corte óptimo y se dice que 4 son diagnósticos (17). Ante lo poco sensible de esta clasificación, en el año 2007, en el Consenso Internacional de Rosemont se propuso una nueva clasificación, en esta hay unos criterios parenquimatosos mayores que son $(14,18)$ :

- Focos hiperecoicos mayores de $2 \mathrm{~mm}$ en longitud y ancho con sombra acústica

- Lobularidad (mayor o igual de 3 lóbulos contiguos como en panal de abejas).

Hay unos criterios menores parenquimatosos que son:

- Focos hiperecoicos (>2 mm de longitud sin sombra acústica)

- Bandas (mayores o iguales de $3 \mathrm{~mm}$, a menos en dos diferentes direcciones)

- Lobularidad (>5 mm no lóbulos contiguos)

- Seudoquiste (anecoico, con o sin septos).

Existen unos criterios ductales:

- Criterio ductal mayor: cálculos del conducto con sombra acústica

- Criterios ductales menores:

- Dilatación del ducto mayor o igual de 3,5 mm en el cuerpo y $1,5 \mathrm{~mm}$ en la cola

- Ducto tortuoso

- Pared de ducto hiperecoica

- Ramas laterales dilatadas.

Se hace diagnóstico con un criterio mayor parenquimatoso y tres menores o con dos criterios mayores parenquimatosos o ductales o con dos criterios mayores parenquimatosos. Se sugiere pancreatitis crónica con tres criterios menores o con solo un parenquimatoso ductal o pancreático $(14,18)$. 
En el año 2010 se realizaron los criterios japoneses para pancreatitis crónica. Esto criterios incluyen (19):

- Hallazgos en las imágenes

- Hallazgos histológicos

- Anormalidad de enzimas pancreáticas en sangre u orina

- Resultados anormales de las pruebas de funcionamiento exocrino.

Esta clasificación japonesa, si bien es más sensible y específica, es difícil de realizar porque combina variables múltiples. Es por esto por lo que cuando usamos la endosonografía es más fácil, sobre criterios de imágenes, tratar de ver los criterios de Rosemont. Algunos de los hallazgos que se pueden observar se resumen de la siguiente forma:

- Calcificaciones o cálculos en ramas secundarias (Figura 1)

- Dilatación del conducto de Wirsung o cálculos dentro de este conducto (Figura 2)

- Bandas fibrosas (Figura 3)

- Dilatación del Wirsung y realce de sus paredes (Figura 4)

- Dilatación de Wirsung, ramas secundarias y lobularidad de la glándula (Figura 5)

- Lobularidad, bandas fibrosas y dilatación de ramas secundarias (Figura 6).

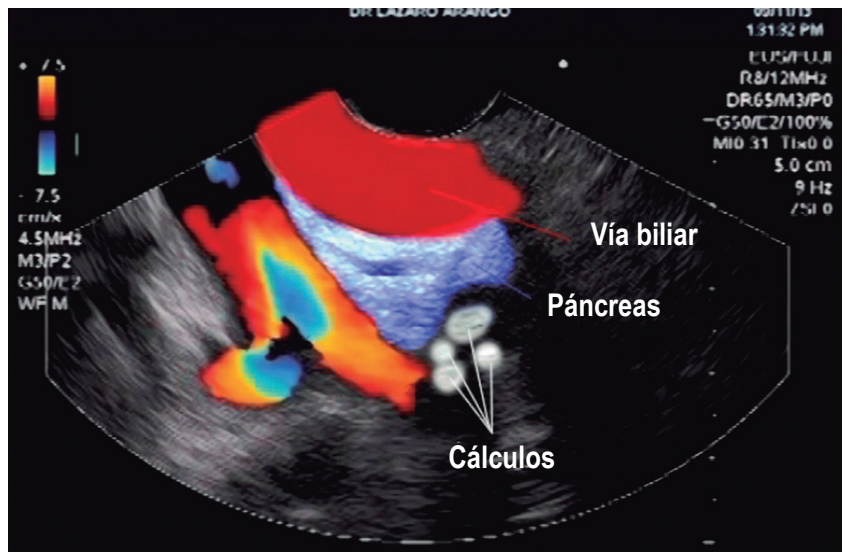

Figura 1. Cálculos en el parénquima del páncreas y ramas vistos por endosonografía lineal de Fujinon (imagen editada por la Unión de Cirujanos SAS, Lázaro Arango).

La USE también integra el uso de elastografía para medir el grado de fibrosis pancreática y su relación con la probabilidad de presentar insuficiencia pancreática exocrina (20).

\section{TRATAMIENTO DE LA PANCREATITIS CRÓNICA}

El tratamiento de la PC consiste en aliviar el dolor, prevenir los ataques recurrentes, corregir las consecuencias de la insuficiencia, tanto endocrina como exocrina (como diabetes o malnutrición), y también tratar las complicaciones que

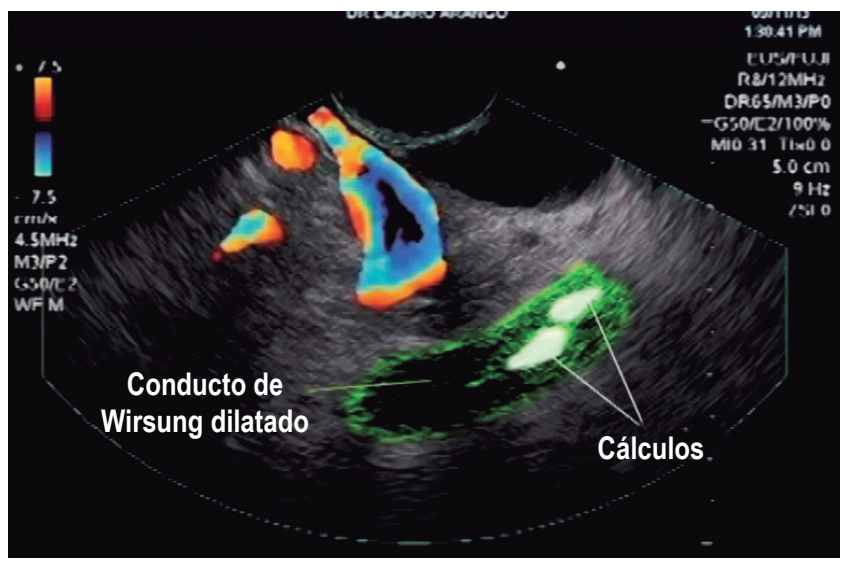

Figura 2. Dilatación del conducto de Wirsung y cálculos dentro de él, vistos con endosonografía lineal de Fujinon (imagen cortesía de la Unión de Cirujanos SAS, Lázaro Arango).

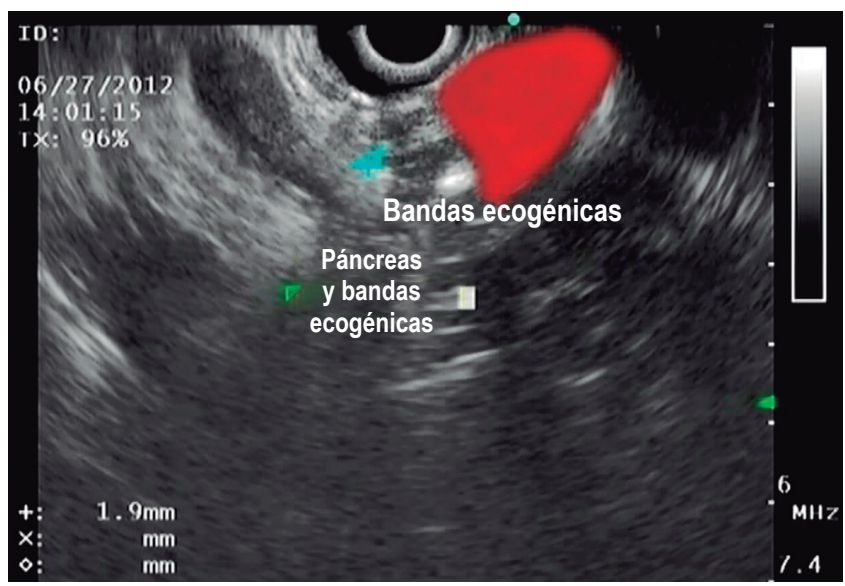

Figura 3. Bandas ecogénicas en endosonografía lineal Olympus (imagen cortesía de la Unión de Cirujanos SAS, Lázaro Arango).

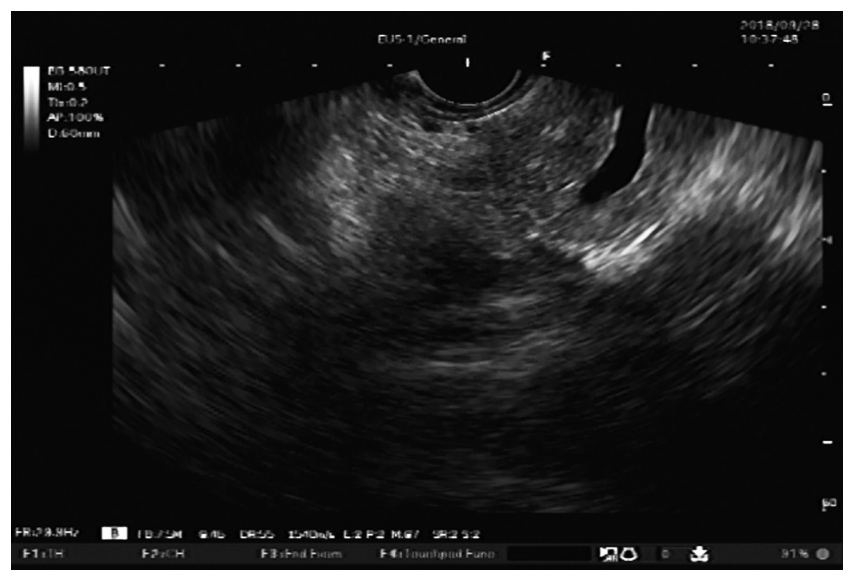

Figura 4. Endosonografía lineal de Fujinon. Dilatación del Wirsung en el cuerpo del páncreas y realce de sus paredes (imagen cortesía de la Unión de Cirujanos SAS, Lázaro Arango). 


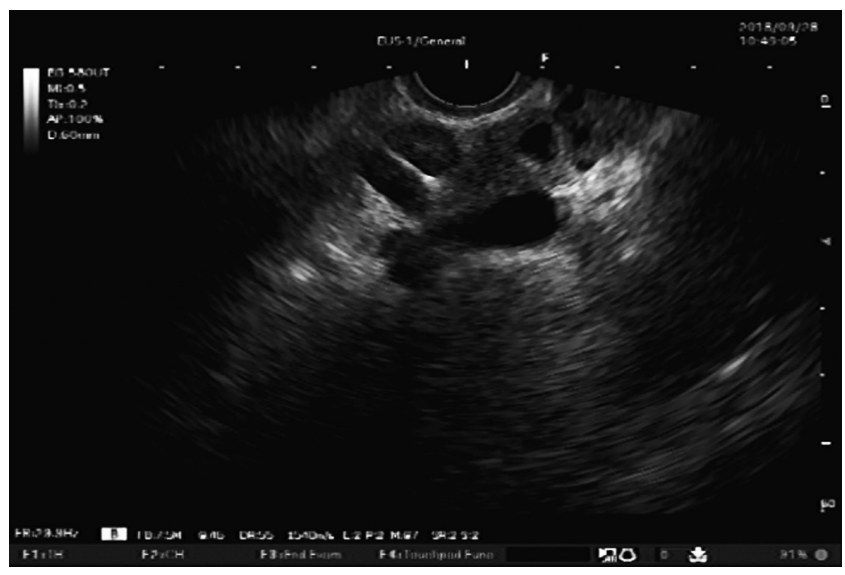

Figura 5. Endosonografía lineal de páncreas con instrumento Fujinon. Dilatación de Wirsung, ramas secundarias y lobularidad de la glándula (imagen cortesía de la Unión de Cirujanos SAS, Lázaro Arango).

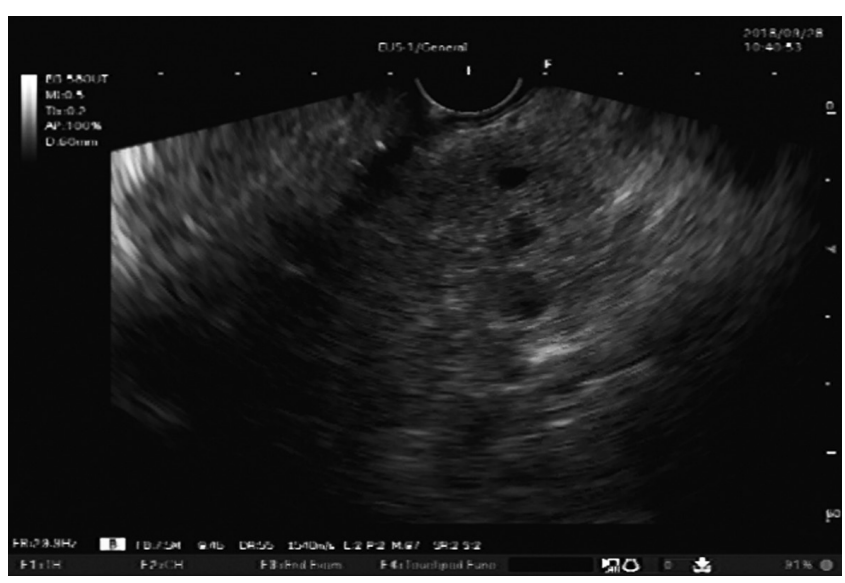

Figura 6. Lobularidad, bandas fibrosas y dilatación de ramas secundarias (imagen cortesía de la Unión de Cirujanos SAS, Lázaro Arango).

puedan surgir. Se inicia con manejo médico, dejando el tratamiento endoscópico, quirúrgico o ambos solo cuando el tratamiento médico adecuado no consigue aliviar el dolor o para el manejo de las complicaciones derivadas de la PC (21).

\section{Tratamiento médico}

El objetivo inicial del manejo médico consiste en la modificación del estilo de vida, suspendiendo el consumo de alcohol y cigarrillo, manejo del dolor y manejo de la insuficiencia pancreática exocrina para evitar el proceso de malnutrición, que lleva a sarcopenia, osteoporosis y aumento de riesgo cardiovascular en estos pacientes (21-24).

En la PC, el dolor es el síntoma inicial en aproximadamente el $75 \%$ de los pacientes. Este está presente durante el curso clínico de la enfermedad en $85 \%-97 \%$ de los pacientes. De ahí la importancia de su manejo. La recomendación para el manejo del dolor es el uso progresivo de la escala analgésica, incrementando la potencia de los medicamentos, según la respuesta, hasta obtener un adecuado control del dolor (25).

El tratamiento de la insuficiencia pancreática exocrina se realiza con enzimas pancreáticas y se indica cuando el paciente presenta esteatorrea mayor de $15 \mathrm{~g} /$ día, pérdida de peso, malabsorción de proteínas o carbohidratos y dispepsia. La dosis de suplencia indicada es $40000 \mathrm{U}$, las cuales se deben administrar durante la ingesta de cada comida principal. Dado que el $\mathrm{pH}$ ácido intraluminal disminuye la acción de la lipasa, se sugiere administrar un inhibidor de bomba de protones en doble dosis para facilitar su acción. La restricción de grasa en la dieta no está indicada, ya que llevaría a ingesta insuficiente de vitaminas liposolubles, las cuales están disminuidas por la propia enfermedad $(1,26)$. Además, se sugiere suplementación de vitaminas liposolubles, como A, D, E, K, vitamina $B_{12}$, micronutrientes y antioxidantes, calcio y vitamina $\mathrm{D}(1)$.

El manejo de la insuficiencia pancreática endocrina (diabetes tipo 3C) es compleja. El medicamento de primera línea usado es la metformina, que se prefiere en pacientes sin malnutrición y con hiperglucemia leve. Algunos pacientes requieren terapia con insulina y se sugiere la supervisión de un endocrinólogo experto (27).

\section{Tratamiento endoscópico}

La terapia endoscópica en la PC está indicada en cálculos que obstruyen el conducto pancreático, estenosis biliares y pancreáticas benignas, drenaje de pseudoquiste pancreático y bloqueo del plexo celíaco (28).

Los cálculos del conducto pancreático principal menores de $5 \mathrm{~mm}$ se manejan con CPRE y maniobras estándar para la extracción de cálculos (Figuras 7-10). Los cálculos mayores de $5 \mathrm{~mm}$ requieren el uso de terapias adicionales con diferentes tipos de litotripsia (electrohidráulica, extracorpórea) y, ahora, el uso de Spyglass, por el que se puede entrar al conducto y aplicar láser y hacer ruptura de los cálculos (29). Nosotros hemos venido aumentando nuestra experiencia con el uso del colangioscopio, y ha sido de mucha utilidad en el manejo no quirúrgico de pacientes con cálculos del páncreas. En las Figuras 11, 12 y 13, se puede observar el Spyglass dentro del conducto de Wirsung, realizando por medio de láser la litotripsia de un gran cálculo. Después del procedimiento se deja siempre un stent de páncreas.

Para el manejo de las estenosis biliares y del conducto pancreático principal se usa la CPRE más esfinterotomía, dilatación con balón y stents (plásticos, metálicos o biodegradables) (30). El manejo del pseudoquiste está indicado cuando se generan síntomas y se puede realizar por vía transpapilar 


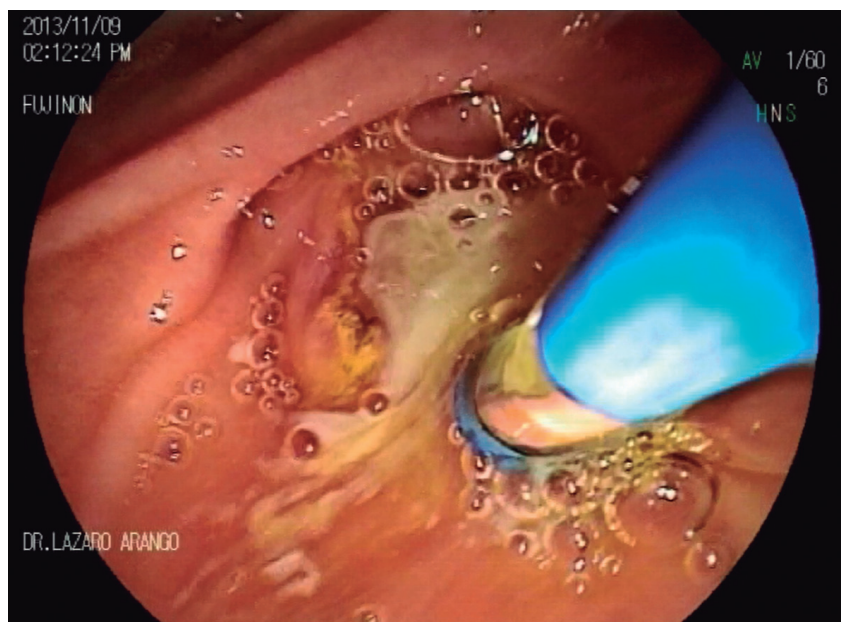

Figura 7. Canulación del conducto de Wirsung. Se observa material turbio por la pancreatitis crónica (imagen cortesía de la Unión de Cirujanos SAS, Lázaro Arango).

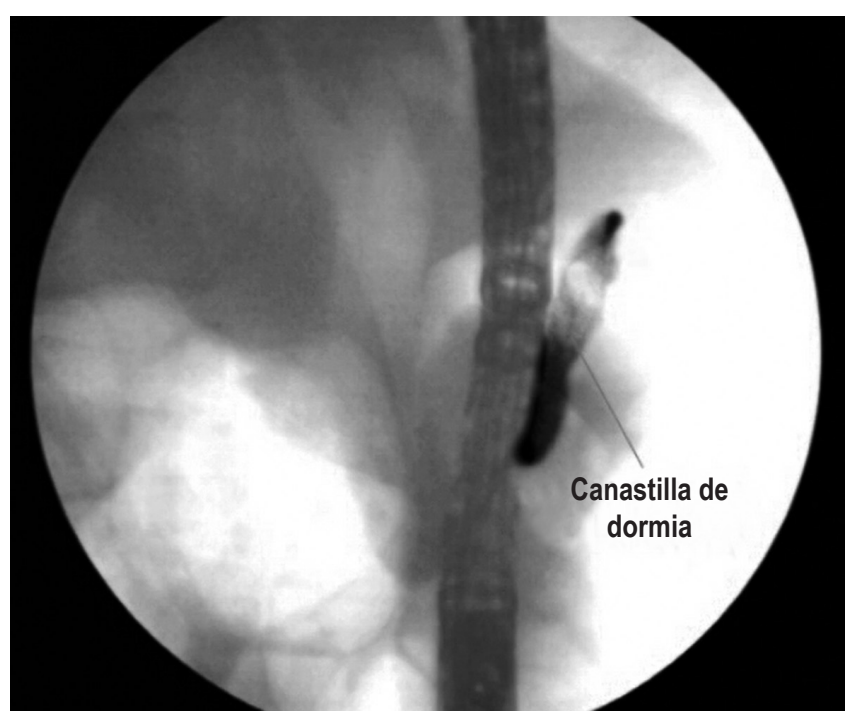

Figura 9. Exploración del Wirsung con canastilla (imagen cortesía de la Unión de Cirujanos SAS, Lázaro Arango).

o transmural (transgástrica o transduodenal). El abordaje guiado por CPRE está indicado para lesiones menores de $5 \mathrm{~mm}$ y asociadas con disrupción del conducto pancreático principal. Para grandes pseudoquistes, el drenaje transmural guiado por USE es el abordaje indicado $(28,30)$.

El bloqueo del plexo celíaco es una medida que se usa para el manejo de dolor (sobre todo en pacientes que requieren altas dosis de narcóticos), lo cual logra un alivio del dolor y un menor consumo de analgésicos. Es de tener en cuenta que su efecto es temporal (21). El procedimiento consiste en la inyección guiada (preferiblemente por USE)

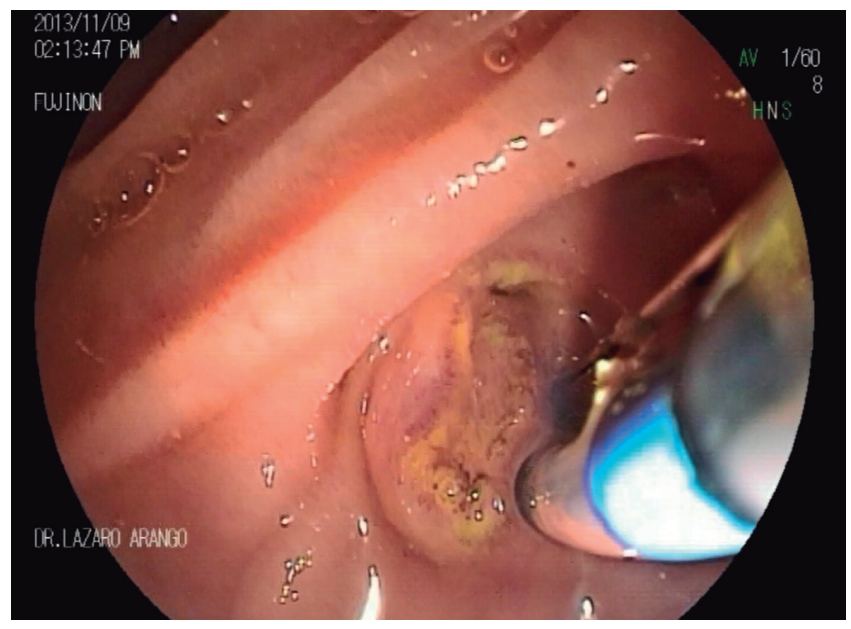

Figura 8. Apertura con corte del ducto pancreático (imagen cortesía de la Unión de Cirujanos SAS, Lázaro Arango).

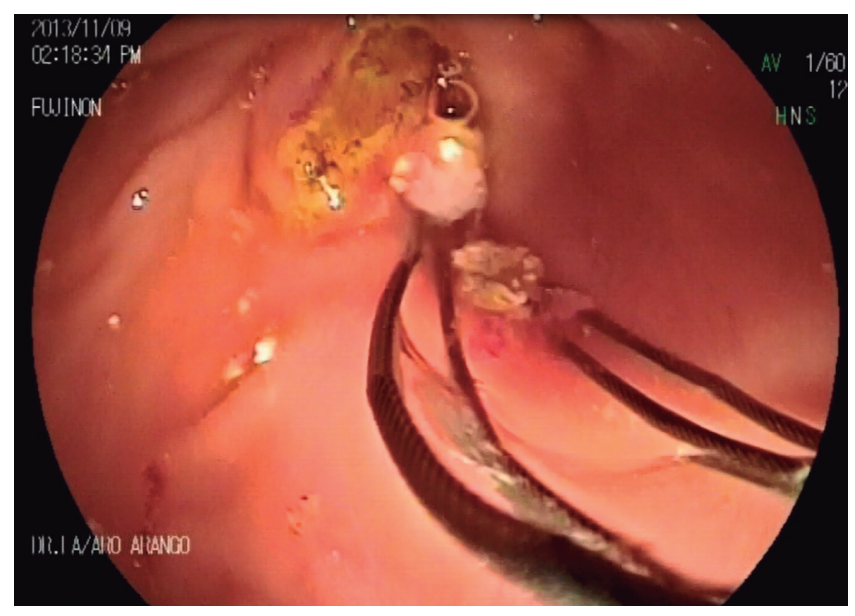

Figura 10. Extracción de cálculos del Wirsung (imagen cortesía de la Unión de Cirujanos SAS, Lázaro Arango).

de esteroide y anestésico local sobre el plexo celíaco, con el fin de cortar las vías aferentes de nocicepción (1).

\section{Tratamiento quirúrgico}

Las intervenciones quirúrgicas en la PC están indicadas cuando el manejo médico y endoscópico de las diferentes complicaciones de la PC han fracasado. Los objetivos de la cirugía son descomprimir los conductos obstruidos y preservar el tejido pancreático. Dentro de los procedimientos quirúrgicos se encuentra la pancreatoyeyunostomía lateral 


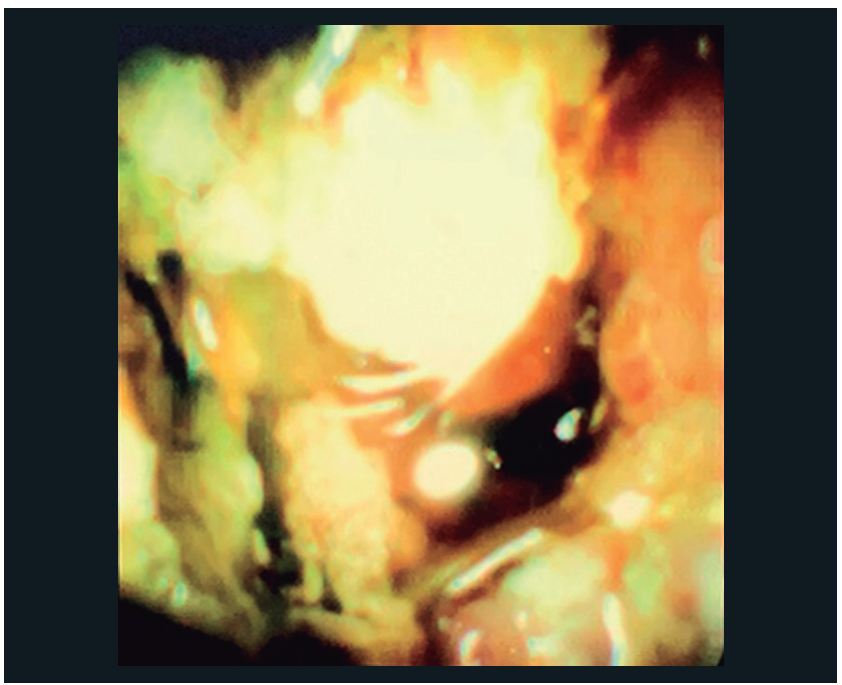

Figura 11. Cálculos dentro del conducto de Wirsung vistos por colangioscopia (imagen cortesía de Unión de Cirujanos SAS, Manizales, Colombia).
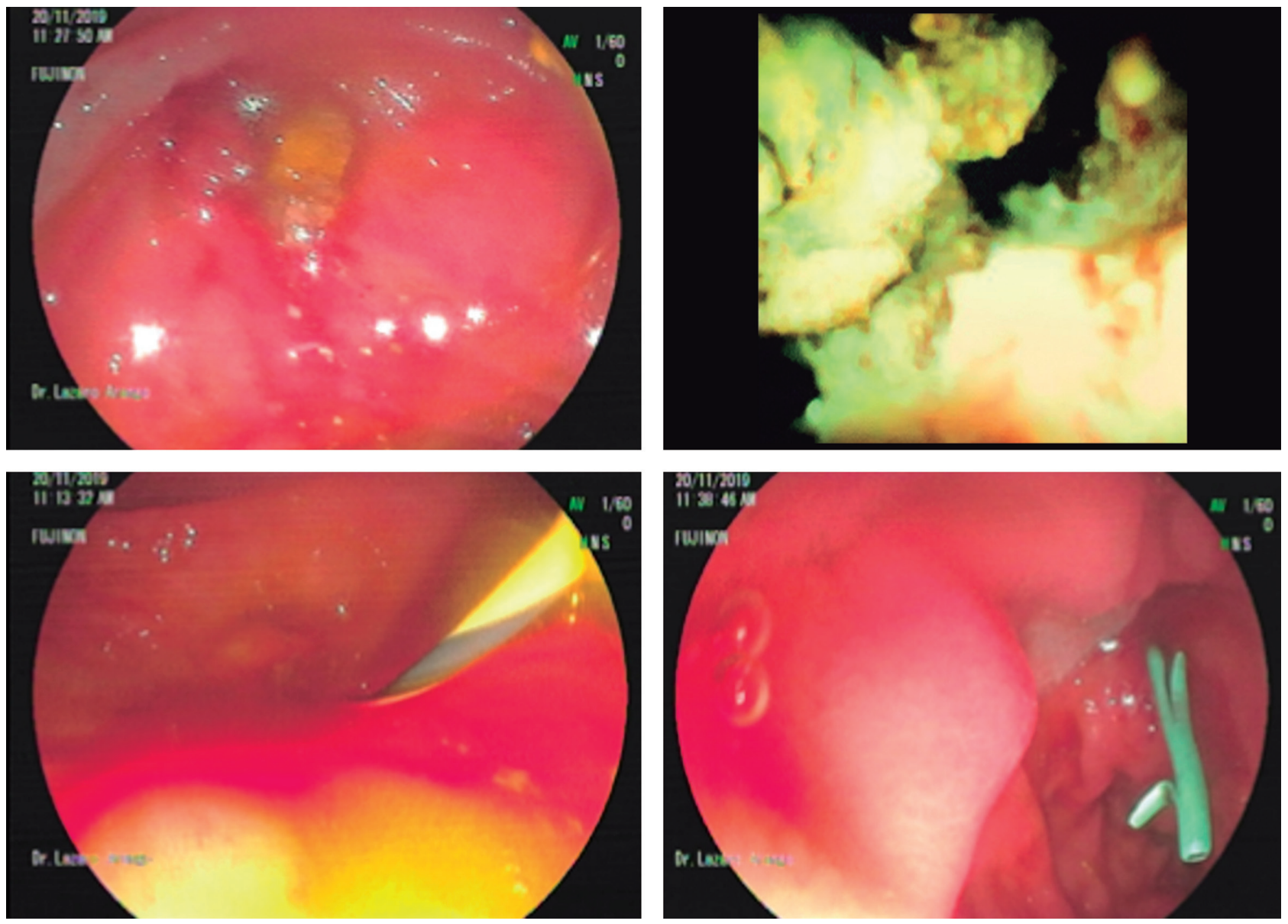

Figura 13. En la parte superior izquierda se observan cálculos que van saliendo con la litotripsia. En la parte superior derecha la misma imagen de la Figura 12. Abajo, a la izquierda, la guía sobre la que introducimos el balón de extracción, para barrer o limpiar los restos de cálculos y a la derecha, el stent de páncreas que siempre se deja al final del procedimiento. 
(Puestow), pancreatectomía parcial y la pancreatectomía total con trasplante autólogo de islotes pancreáticos (21).

\section{CONCLUSIÓN}

La PC es una entidad que genera alteración importante en la calidad de vida de los pacientes que la padecen. El manejo debe estar enfocado inicialmente en el manejo del dolor, pero también en prevenir y manejar las complicaciones asociadas con esta patología. El enfoque debe ser completo y multidisciplinario, con el uso juicioso de terapias de reemplazo e intervenciones tempranas, utilizando las terapias endoscópicas y quirúrgicas en pacientes seleccionados.

\section{Agradecimiento}

Agradecemos al Dr. Camilo Ángel Rodríguez por su colaboración en el seguimiento de los pacientes sometidos a Spyglass del páncreas por cálculos y en la revisión de la literatura pertinente de índices de riesgo.

\section{REFERENCIAS}

1. Trikudanathan G, Navaneethan U, Swaroop S. Modern treatment of patients with chronic pancreatitis, Gastroenterol Clin N Am. 2012;41:63-76. doi: https://doi.org/10.1016/j. gtc.2011.12.009.

2. Lizarazo J. Fisiopatología de la pancreatitis crónica. Rev Col Gastroenterol. 2008; (3):290-8.

3. Yang D, Forsmark C. Chronic pancreatitis. Curr Opin Gastroenterol. 2017;33:396-403. doi: https://doi. org/10.1097/MOG.0000000000000377.

4. Siddiqui U, Hawes R. Chronic pancreatitis. En: Chandrasekhara V, Khashab M, Elmunzer BJ, Muthusamy VR. Clinical Gastrointestinal Endoscopy. 3a edición. Elsevier. 2019. p. 68698. doi: https://doi.org/10.1016/C2014-0-03901-9.

5. Anaizi A, Hart P, Conwell D. Diagnosing chronic pancreatitis. Dig Dis Sci. 2017;62(7):1713-20. doi: https://doi. org/10.1007/s10620-017-4493-2.

6. Nikfarjam M, Wilson J, Smith R, Australasian Pancreatic Club Pancreatic Enzyme Replacement Therapy Guidelines Working Group. Diagnosis and management of pancreatic exocrine insufficiency. Med J Aust. 2017;207(4):161-5. doi: https://doi.org/10.5694/mja16.00851.

7. Conwell D, Lee L, Yadav D, Longnecker DS, Miller FH, Mortele KJ, et al. American Pancreatic Association Practice Guidelines in Chronic Pancreatitis: evidence-based report on diagnostic guidelines. Pancreas. 2014;43(8):1143-62. doi: https://doi.org/10.1097/MPA.0000000000000237.

8. Vanga RR, Tansel A, Sidiq S, El-Serag HB, Othman MO, et al. Diagnostic performance of measurement of fecal elastase-1 in detection of exocrine pancreatic insufficiency - systematic review and meta-analysis. Clin Gastroenterol Hepatol. 2018;16(8):1220-8.e4. doi: 10.1016/j.cgh.2018.01.027.
9. Domínguez-Muñoz JE, Nieto L, Vilarino M, Lourido MV, Iglesias-García J, et al. Development and diagnostic accuracy of a breath test for pancreatic exocrine insufficiency in chronic pancreatitis. Pancreas. 2016;45:241-7. doi: https:// doi.org/10.1097/MPA.0000000000000434.

10. Frøkjær JB, Akisik F, Farooq A, Akpinar B, Dasyam A, Drewes AM, et al. Guidelines for the diagnostic cross sectional imaging and severity scoring of chronic pancreatitis. Pancreatology. 2018;18(7):764-73. doi: 10.1016/j. pan.2018.08.012.

11. Tirkes T. Chronic pancreatitis what the clinician wants to know from MR Imaging, Magn Reson Imaging Clin N Am. 2018;26(3):451-61. doi: https://doi.org/10.1016/j. mric.2018.03.012.

12. Irisawa A, Shibukawa G, Abe Y, Nikaido A, Inbe K, Hoshi K. Endosonographic diagnosis of chronic pancreatitis. J Gastroint Dig Syst. 2013;S2:005. doi: https://doi. org/10.4172/2161-069X.S2-005.

13. Cañadas R, Aponte D, Albis R, Sabbagh LC. Utilidad del ultrasonido endoscópico en el diagnóstico de pancreatitis crónica: la importancia de un diagnóstico temprano. Rev Col Gastroenterol. 2013;28(3):226-34.

14. Arango L, Diaz C. Ultrasonografía endoscópica en patologías del páncreas. Rev Med Clin Las Cond. 2015;(5):556-710.

15. Sabbagh L, Aponte D, Cañadas R, Torres M, Álvarez E, Prieto RG, et al. Guía de práctica clínica para el uso de ultrasonido endoscópico en pancreatitis crónica, lesiones quísticas y sólidas del páncreas en adultos. Rev Col Gastroenterol. 2015;(11):97-104.

16. Sheel A, Baron R, Sarantitis I, Ramesh J, Ghaneh P, Raraty MGT, et al. The diagnostic value of Rosemont and Japanese diagnostic criteria for 'indeterminate, 'suggestive,' 'possible' and 'early' chronic pancreatitis. Pancreatology. 2018;18(7):774-84. doi: https://doi.org/10.1016/j.pan.2018.08.002.

17. Witt H, Apte MV, Keim, Wilson JS. Chronic pancreatitis: challenges and advances in pathogenesis, genetics, diagnosis, and therapy. Gastroenterology. 2007;132:1557-73. doi: https://doi.org/10.1053/j.gastro.2007.03.001.

18. Catalano M, Sahai A, Levy M, Romagnuolo J, Wiersema M, Brugge W, et al. EUS-based criteria for the diagnosis of chronic pancreatitis: the Rosemont classification. Gastrointes Endos. 2009;(7):1251-61. doi: https://doi.org/10.1016/j. gie.2008.07.043.

19. Shimosegawa T, Kataoka K, Kamisawa T, Miyakawa H, Ohara $\mathrm{H}$, Ito $\mathrm{T}$, et al. The revised Japanese clinical diagnostic criteria for chronic pancreatitis. J Gastroenterol. 2010;45:584-91. doi: https://doi.org/10.1007/s00535-010-0242-4.

20. Domínguez-Muñoz J, Iglesias-García J, Castiñeira Alvariño M, Luaces Regueira M, Lariño-Noia J. EUS elastography to predict pancreatic exocrine insufficiency in patients with chronic pancreatitis, Gastrointest Endosc. 2015;81:136-42. doi: https://doi.org/10.1016/j.gie.2014.06.040.

21. Czul F, Coronel E, Done JA. Una actualización de pancreatitis crónica: artículo de revisión. Rev Gastroenterol Perú. 2017;37(2):146-55. 
22. Han S, Kheder J, Bocelli L, Fahed J, Wachholtz A, Seward G, et al. Smoking cessation in a chronic pancreatitis population. Pancreas. 2016;45:1303-8. doi: https://doi.org/10.1097/ MPA.0000000000000641.

23. Duggan S. Negotiating the complexities of exocrine and endocrine dysfunction in chronic pancreatitis. Proceedings Nutrition Soc. 2017;76:484-94. doi: 10.1017/ S0029665117001045.

24. De la Iglesia D, Vallejo-Senra N, López-López A, IglesiasGarcia J, Lariño-Noia J, Nieto-García L, et al. Pancreatic exocrine insufficiency and cardiovascular risk in patients with chronic pancreatitis (CP). A prospective, longitudinal cohort study. J Gastroenterol Hepatol. 2019;34(1):277283. doi: https://doi.org/10.1111/jgh.14460.

25. Drewes AM, Bouwense S, Campbell CM, Ceyhand GO, Delhaye M, Demir IE, et al. Guidelines for the understanding and management of pain in chronic pancreatitis,
Pancreatology. 2017;17(5):720-31. doi: https://doi. org/10.1016/j.pan.2017.07.006.

26. Domínguez J. Fisiopatología, diagnóstico y tratamiento de la insuficiencia pancreática exocrina en el paciente con pancreatitis crónica. Gastroenterol Hepatol. 2005;28(2):22-8. doi: https://doi.org/10.1157/13071383.

27. Majumder S, Chari ST. Chronic pancreatitis. Lancet. 2016;387(10031):1957-66. doi: 10.1016/S01406736(16)00097-0.

28. Adler J, Gardner T. Endoscopic therapies for chronic pancreatitis. Dig Dis Sci. 2017;1729-37. doi: https://doi. org/10.1007/s10620-017-4502-5.

29. Derdeyn J, Laleman W. Current role of endoscopic cholangioscopy. Curr op Gastroentrol. 2018;34(5):301-8. doi: 10.1097/MOG.0000000000000457.

30. Moran R, Elmunzer B. Endoscopic treatment of pain in chronic pancreatitis. Curr Opin Gastroenterol. 2018;34(6):46976. doi: 10.1097/MOG.0000000000000480. 